\title{
An Approach for Computing Sentiment Polarity Analysis of Complex Why-type Questions on Product Review Sites
}

\author{
Amit Mishra and Sanjay Kumar Jain \\ Computer Engineering Department, NIT Kurukshetra, Haryana, \\ India \\ amitmishrag@gmail.com,skj_nith@yahoo.com
}

\begin{abstract}
Opinion questions expect answers from opinionated data available on social web. Opinion why-questions require answers to include reasons, elaborations, explanations for the users' sentiments expressed in the questions. Sentiment analysis has been recently used in answering why type opinion questions. In this paper, we propose an approach to determine the sentiment polarity of complex why type opinion questions that could be expressed in multiple sentences or could have mixed opinions expressed in them. We apply Rhetorical structure theory to determine discourse structure of why type questions. We use such structure to determine sentiment polarity of why type questions and conduct experiments which obtain better results as compared to baseline average scoring methods.
\end{abstract}

Keywords: Question answering, information retrieval, natural language processing, natural language understanding and reasoning.

\section{Introduction}

Question Answering Systems (QASs) provide specific answers to users' questions. Most of the research related to Why-type questions in QASs consults information source based on facts i.e., newspaper, technical documents etc [2, 25, 26]. Such questions ask for some facts or methods e.g., why Roses are red? With the emergence of Web 2.0, there are massive user generated data on the web such as social networking sites, blogs, review sites, etc. [23]. These opinionated data sources contain public opinions which could help the users in making judgment about the products. Hence, they could contain answers to why-type questions such as why should I look for product $\mathrm{x}$ ? $[1,4,5,6]$. Such questions are referred as opinion questions $[1,4]$. The task of generating answers to these questions requires application of opinion mining techniques along with Natural language processing techniques $[1,2,4]$. Research related to why-opinion questions consider simple whyquestions expressed in single sentence $[1,2,4,5,6]$. To the best of our knowledge there is no work on complex why-type questions that could be expressed in multiple sentences or could have mixed opinions expressed in them. From literature $[1,2,4,5$, 6], we find that determining the sentiment polarity of why-questions is a significant phase for generating correct answers as it searches for intention of users 
with which he is looking for products. Such analysis would determine type of public comments (positive or negative) required to be presented as answers. Most researchers follow average scoring methods which compute the average scores of words in order to determine the final sentiment scores of objects for the task of opinion mining $[3,4,5,11,12,13,24]$.

Such average scoring methods could fell flat in real life scenario for opinion mining tasks [29, 30, 31, 32, 33]. Average scoring methods could yield inaccurate results when applied on complex why-type questions e.g., "I need mobile with good camera. Why Nokia is a bad choice?" Another example, why movie X is bad even if brad has delivered good performance? The average scoring approach could yield false results in determining sentiment polarity of such questions as "bad" and "good" opinion words will neutralize each other to assign neutral score to questions in terms of sentiment polarity. In such circumstances, there is a natural need to fragment why- question into more important and less important spans in view of opinion mining. In the above example, the overall intention of user is determined through text span "Why movie X is bad" not through "brad has delivered good performance".

Bas Heerschop et al. state that most research done in field of sentimental analysis do not take account of documents important structural feature [25]. The authors use rhetorical structure theory to determine discourse structure of document to perform document level sentiment analysis which gives promising results.

Ziheng Lin et al. state that their discourse parser could be utilized in generating answers to why-questions by recognizing causal relations in text [21]. This motivates us to perform discourse based analysis of why-questions.

We perform discourse based analysis of why-questions through a PDTB-Styled End-to-End Discourse Parser developed by Ziheng Lin et al. [21]. We fragment questions into different text spans i.e. more important and less important spans for opinion mining. We use this relation further to determine sentiment polarity of Why-questions. From literature, we find that SentiWordNet [9], MPQA [7], WordNet [15], and Bing Liu's Opinion Lexicon [19] lexical resources are extensively used in opinion mining.

Most of the words are either absent or having stronger objective scores (neutral scores) in these lexical resources [3]. Such words could behave as opinion words when used in questions. For example, why should I choose the product? Here all the words are strong objective words based on SentiWord Net, MPQA, and Bing Liu's Opinion Lexicon. The existing average scoring methods will classify questions as neutral but it asks for positive opinions about the product. Hence, for the task of sentiment classification, the recompilation of the score is necessary in order to determine correct polarity of why-questions.

We present an approach for finding sentiment polarity of complex opinion whyquestions. The complex why type- opinion questions could be expressed in multiple sentences or could have mixed opinions expressed in questions. In summary our contribution is as follows:

1. We fragment why-questions into more important and less important spans using a discourse parser [21] and compute score of whyquestions as positive, or negative or neutral on the basis of sentiment scores of words of questions computed using different lexical resources. 
An Approach for Computing Sentiment Polarity Analysis of Complex Why-type Questions ...

2. We propose an algorithm which re-computes sentiment polarity scores of different spans of question and perform better in comparison to baseline average scoring methods [2, 4, 5, and 6] in determining opinion polarity of why-questions.

Rest of the paper is organized as follows Section 2 discuss related work. Section 3 presents our Approach for determining sentiment polarity of Whyquestions. We have results and discussion in Section 4. Finally, we have conclusions and scope for future research in Section 5.

\section{Related Work}

Based on works on opinion question answering $[1,2,4,5,6]$, we find that question analysis, document analysis, retrieval method and answer processing are the steps in drawing answers to opinion why questions. Output of the question analysis phase has cascade effects on other phases in generating correct answers. Further, we find that question analysis comprises of several sub processes i.e., recognizing entity in question, identifying its aspects, detecting sentiment polarity of question and question form. Determining polarity of why-questions is a significant phase for generating correct answers as it searches for intention of users expressed in questions related to products. Sentiment polarity of opinion questions is determined through identification of opinion bearing words and computation of their polarity score through opinion lexical resources $[1,2,4,5,6]$. S Moghaddam et al develop an opinion question answering system in which they consider only adjectives as opinion bearing words for the task of determining sentiment polarity of questions [4, 8]. They use a subset of seed words containing 1,336 adjectives. These words are manually classified into 657 positives and 679 negatives by Hat Zivassiloglov et al. [14]. In another work, Farah Benamara found that adjectives and adverbs work better than adjectives alone for the task of sentiment polarity detection [16]. Muhammad Abuliash et al. use adjectives or adjectives headed by adverbs as opinion bearing words in text documents to produce summary of review documents on the basis of features through semantic and linguistic analysis using SentiWordNet [13]. These researchers ignore nouns and verbs which could also behave as opinion words. Turney found that adjectives, verbs, nouns and adverbs play significant role as opinion bearing words for the task of opinion mining [17]. Jong $\mathrm{Hu}$ et al. consult a Japanese polarity dictionary distributed via Alagin forum in their question answering [2]. The dictionary is not available in English. Jianxing Yu et al. present an opinion question answering system for products review sites by exploiting hierarchical organization of the product reviews [5]. They use SVM sentiment classifier to determine sentiment polarity of questions. For doing this, they consult the MPQA project sentiment lexicon. Most of the words in MPQA project are objective words such as buy; purchase, choose etc. hence we consider the corpus as not a good choice. SenticNet detect sentiment polarity of single sentence by using machine-learning and knowledge-based techniques [29, 30, 31, 32, 33]. The SenticNet capture the conceptual and affective information in the sentence by using 
the bag-of- concepts model. The system assumes that input text is opinionated. It does not deal with multiple sentences.

Hongping $\mathrm{Fu}$ et al. classify opinion questions into 8 classes: holder, sentiment, target, reason, comparison, $\mathrm{y} / \mathrm{n}$, time and location. With regard to why-questions, opinion why-questions could be divided into two classes: open why- questions (Why-questions with unknown reason) and closed why-questions (Why-questions with reason selection) [1]. Fan Bu classify why questions as questions requiring explanations or opinions of others [22].

\section{Proposed Approach}

In this section, we determine sentiment polarity of why-questions in order to determine the intention of the users with which they are looking for products. We fragment complex why- questions into more important and less important spans in view of opinion mining and then compute sentiment polarity of why-questions on the basis of polarity of more important text span.

\subsection{Segmentation of Why-questions in View for Opinion Mining}

The objective of this fragmentation is to fragment questions into different text spans and categorize them into more important and less important text span for opinion mining of questions. We present the algorithm that fragment why- questions into more important and less important spans using a discourse parser [21]. The algorithm is as follows:

1. The question text span is parsed through A PDTB-Styled End-to-End Discourse Parser developed by Ziheng Lin et al. [21]

2. If relation equals Cause, or Conjunction or Contrast choose $\operatorname{Arg}(2)$ span as first priority.

3. Else If relation equals Condition or others, then choose $\operatorname{Arg}(1)$ span as first priority. The output of this algorithm will be a number of text spans with different priorities.

Example:

"I need a mobile with good sound quality and nice looks. I went to market. I found three good shops. I went to shop number 3 . Why should one feel sad finally?"

We see the output file as shown below:

\{NonExp_0_Arg1 \{NonExp_0_Arg1 I need a mobile with good sound quality and nice looks. NonExp_0_Arg1\} NonExp_0_Arg1\}\{NonExp_1_Arg1 \{NonExp_0_Arg2_EntRel \{NonExp_1_Arg1 \{NonExp_0_Arg2_EntRel I went to market. NonExp_0_Arg2\} NonExp_1_Arg1\} NonExp_0_Arg2\} NonExp_1_Arg1\} \{NonExp_2_Arg1 \{NonExp_1_Arg2_EntRel \{NonExp_2_Arg1 \{NonExp_1_Arg2_EntRel I found three good shops. NonExp_1_Arg2\} NonExp_2_Arg1\} NonExp_1_Arg2\} NonExp_2_Arg1\} \{NonExp_3_Arg1 \{NonExp_2_Arg2_EntRel \{NonExp_3_Arg1 \{NonExp_2_Arg2_EntRel I went to 
shop number 3. NonExp_2_Arg2\} NonExp_3_Arg1\} NonExp_2_Arg2\} NonExp_3_Arg1\}\{NonExp_3_Arg2_Cause \{NonExp_3_Arg2_Cause Why should one feel sad finally? NonExp_3_Arg2 \} NonExp_3_Arg2\}

For relation Non Exp 3 cause, we see Arg 1 as "I went to shop number 3", and Arg 2 as "Why should one feel sad finally?". Hence we select Arg 2 as more important text span. Hence the overall intention of user with which he is looking for product is expressed in Arg 2 text span "Why should one feel finally?"

\subsection{Computation of Sentiment Polarity of why-questions}

Polarity of why-questions. We compute sentiment polarity of why-questions through the analysis of more important text span of question and determine the scores on the basis of sentiment scores of opinion words of the text span $[1,2,4,6]$. From literature surveyed, we find that adjectives, nouns, adverb, verb could behave as opinion bearing words. In this regard, we parse the question text span through the Stanford Parser [10] to determine the part of speech of each word. We remove Pre compiled Stopwords from the question words to get opinion words. We change opinion words to their root form through morphological analysis.

We classify the sentiment polarity (i.e. Positive, or negative or neutral) of Question text span through following steps as discussed below:

Computing score of Opinion word: we compute the score of each opinion word of question text span through methods described in literature using different popular sentiment lexicons ie, SentiWord Net, MPQA, Word Net, Bing Liu Opinion lexicon. We propose a method which performs better in comparison to the discussed methods.

Computing score of Question text span (Question Polarity Scoring (QPS)): We take average of scores of words to determine overall sentiment polarity of question text span $[5,7,8,10,12$, and 28].

3.2.1 Computing score of Opinion word [10, 12, 28]. In this section, we compute the score of each opinion word of question text span by using different popular opinion lexicons, i.e., SentiWord Net, Bing Liu opinion lexicon, MPQA and Word Net. We recomputed the score of words through our algorithm.

Scoring Method 1 consulting SentiWordNet [11, 12, 20]. SentiWordNet is a dictionary of words where scores (positive, negative or neutral) are assigned in the range 0 to 1 to each synset of WordNet.

We compute score of each opinion word through method discussed in papers [11, $12,20]$. Each tokenized word with determined part of speech in the question text span is allotted a positive or negative score with the help of SentiWordNet. As there could be a number of synsets of the word, the score of word is computed as the average score of all synsets of that word.

The positive score is computed as the average of the positive scores of all the synsets corresponding to that word available in SentiWordNet which have same part of speech as of question text span word. Same is done for calculating negative score. Those words which are not found in SentiWordNetare assigned zero.

WordScore(w) is computed by averaging the score (both positive and negative) of the individual words present in the question text span related to the feature M: 


$$
\text { WordScore }(W)=\frac{1}{n} \sum_{i=1}^{n} \text { posScore }(i)+\frac{1}{n}\left(-\sum_{i=1}^{n} \text { negScore }(i)\right)
$$

where posScore(i), negScore(i) are the positive, or negative score respectively found as of i-th synset of word in question text span S. $n=$ Total Number ofsynsets of word.

As there are $93.75 \%$ of words in SentiWordNetare having stronger objective score [3]. Also most of the words have zero positive and negative score such as choose etc. Hence there is need to recomputed the score of such words.

Scoring Method 2 consulting WordNet $[15,18]$. We used the Sentiment Symposium Tutorial: Lexicons, prepared by Christopher Potts of Stanford Linguistics for computing the score of a word [18]. WordNet is used here [15]. The WordScore $(\mathrm{w})$ is computed by averaging the score of the individual words $(\mathrm{w})$ present in the question text span related to the feature M:

$$
\text { WordScore }(w)=\frac{1}{n} \sum_{i=1}^{n} \operatorname{mws}(i)
$$

Scoring Method 3 using OpinionFinder [28]. We perform subjectivity analysis of Why questions using OpinionFinder System. Opinion Finder recognizes subjective sentences as well as different aspects of subjectivity within sentences.

Scoring Method 4 consulting Bing Liu Opinion Lexicon. We used Bing Liu Opinion Lexicon prepared by Bing Liu [19]. It provides list of positive words and negative words. It does not contain ambiguous words. Hence the coverage is very low with only 2006 number of Positive words and 4783 number of Negative words. If the number of positive words in Question text span is more than number of negative words, then we classify it as Positive else negative.

Our Method: Our modified Word Scoring methods. In our approach, we search for synonymous words to improve the sentiment polarity of why-questions. From our experiments, we find that MPQA and SentiWordnet is the effective dictionary for the purpose. Our approach is as follows:

1. Calculate score of each argument.

2. We compute the score of opinion word extracted in section 3.2. We calculate the score of the word through following rules. As there are two values for subjective score (strong or weak), and two values of positive score (strong or weak) and two values of negative scores (strong or weak) hence there are $(2 * 2 * 2=8)$ combinations. And there is one combination of words not found in corpus. Each word score in each argument is calculated from MPQA dictionary

3. If the polarity of word is positive or negative regardless of its score and strength is strongsubj or weaksubj. Then, final score of word will be made same.

- Strong positive with strong subj of word has score equivalent to 1.00 .

- Strong positive with weak subj of word has score equivalent to .75 . 
- Weak positive with strong subj of word has score equivalent to .50 .

- Weak positive with weak subj of word has score equivalent to .25 .

- The word which is not found in the corpus is assigned score 0.00 .

- Weak negative with weak subj of word has score equivalent to -0.25 .

- Weak negative with strong subj of word has score equivalent to -0.50 .

- Strong negative with weak subj of word has score equivalent to -0.75 .

- Strong negative with strong subj of word has score equivalent to -1.00 .

4. Else the score of the word is calculated with the help of SentiWord Net.

We update Scoring Method 1 consulting SentiWordNet. We do some extra computation on WordScore(w) if it equals to zero. We compute WordScore(w). If WordScore(w) equals to zero, then we search for other synonymous words falling in same synonymous set. We compute WordScore(w)

For example: if I need average mobile, why should I choose the product $X$ ?, Choose is synonymous with take\#10, select\#1, pick_out\#1, prefer\#2 opt\#1 in sentiWord Net. Hence the updated positive score of the "choose" word is average sum of all positive scores of synonymous words. Same is done for negative score computation.

3.2.2 Computing score of Question text span (Question Polarity Scoring (QPS)). QPS is computed by averaging the score (both positive and negative) of the opinion words present in the question text span related to the feature M:

$$
\operatorname{QScore}(q)=\frac{1}{n} \sum_{i=1}^{n} \text { WordScore }(i)
$$

where QScore $(\mathrm{q})$ score of question text span $\mathrm{Q}$ which is related to product feature $\mathrm{M}$. WordScore(i) is score found of ith word (w) in question text span S. $\mathrm{n}=$ Total Number ofwords in Question text span. Based on value of QScore(q), we determine polarity of question span text q. If QScore(q) is positive, hence question span text $\mathrm{q}$ have positive polarity. QScore $(\mathrm{q})$ is negative, hence question span text $\mathrm{q}$ have negative polarity. QScore(q) is neutral, hence question span text $\mathrm{q}$ is neutral.

We analyze 19 manually constructed opinion why-questions with different structures prepared by our colleagues that could be asked on product review sites [the list of questions are given after reference section]. There is no standard data set for opinion "why" questions to the best of our knowledge. We find accuracy of Question Fragmentation module for opinion mining in Table 1. The details are given after reference section. We do the analysis of the questions and determine their sentiment polarity. We followed evaluation method of authors S. Moghaddam et al. in Table 2 [4]. We do analysis of list of questions and their sentiment polarity detection in Table 3. In Table 3, we present the accuracy observed in different methods. 
Table 1. Accuracy of Question Fragmentation module for opinion mining [4].

\begin{tabular}{|l|c|}
\hline Method & Our Method \\
\hline Accuracy & $60 \%$ \\
\hline
\end{tabular}

Table 2. Analysis of sentiment polarity of 'more important text span' of questions [4].

\begin{tabular}{|c|c|c|c|c|c|c|}
\hline & Questions & $\begin{array}{c}\text { Met } \\
\text { hod } 1\end{array}$ & $\begin{array}{c}\text { Met } \\
\operatorname{hod} 2\end{array}$ & $\begin{array}{l}\text { Meth } \\
\text { od } 3\end{array}$ & $\begin{array}{c}\text { Met } \\
\text { hod } 4\end{array}$ & $\begin{array}{r}\text { Our } \\
\text { method }\end{array}$ \\
\hline 1. & Why should I buy Nokia? & $\sqrt{ }$ & $\sqrt{ }$ & $\mathrm{X}$ & $\sqrt{ }$ & $\sqrt{ }$ \\
\hline 2. & Why should I like Nokia? & $\sqrt{ }$ & $\sqrt{ }$ & $\sqrt{ }$ & $\sqrt{ }$ & $\sqrt{ }$ \\
\hline 3. & Why should I go for Nokia? & $\sqrt{ }$ & $\mathrm{x}$ & $\mathrm{x}$ & $\mathrm{x}$ & $\sqrt{ }$ \\
\hline 4. & Why should I look for Nokia? & $\sqrt{ }$ & $\mathrm{X}$ & $\mathrm{x}$ & $\mathrm{x}$ & $\sqrt{ }$ \\
\hline 5. & Why should I accept Nokia? & $\sqrt{ }$ & $\sqrt{ }$ & $\sqrt{ }$ & $\mathrm{x}$ & $\sqrt{ }$ \\
\hline 6. & Why should I choose Nokia? & $\mathrm{x}$ & $\mathrm{x}$ & $\mathrm{x}$ & $\mathrm{x}$ & $\sqrt{ }$ \\
\hline 7. & Why should I forget Nokia? & $\sqrt{ }$ & $\mathrm{x}$ & $\sqrt{ }$ & $\mathrm{x}$ & $\sqrt{ }$ \\
\hline 8. & $\begin{array}{l}\text { Why should I get fond of } \\
\text { Nokia? }\end{array}$ & $\sqrt{ }$ & $\mathrm{x}$ & $\sqrt{ }$ & $\sqrt{ }$ & $\sqrt{ }$ \\
\hline 9. & Why should I overlook Nokia? & $\sqrt{ }$ & $\sqrt{ }$ & $\sqrt{ }$ & $\sqrt{ }$ & $\sqrt{ }$ \\
\hline 10. & Why should I suggest Nokia? & $\sqrt{ }$ & $\mathrm{x}$ & $\sqrt{ }$ & $\mathrm{x}$ & $\sqrt{ }$ \\
\hline 11. & $\begin{array}{c}\text { Why should I recommend } \\
\text { Nokia? }\end{array}$ & $\sqrt{ }$ & $\mathrm{x}$ & $\sqrt{ }$ & $\sqrt{ }$ & $\sqrt{ }$ \\
\hline 12. & Why should I propose Nokia? & $\mathrm{x}$ & $\mathrm{x}$ & $\mathrm{x}$ & $\mathrm{x}$ & $\sqrt{ }$ \\
\hline 13. & Why should I advise for Nokia? & $\mathrm{x}$ & $\mathrm{x}$ & $\sqrt{ }$ & $\mathrm{x}$ & $\sqrt{ }$ \\
\hline 14. & Why should I need Nokia? & $\mathrm{x}$ & $\mathrm{x}$ & $\mathrm{x}$ & $\mathrm{x}$ & $\mathrm{X}$ \\
\hline 15. & Why should I feel sad? & $\sqrt{ }$ & $\sqrt{ }$ & $\mathrm{x}$ & $\mathrm{x}$ & $\sqrt{ }$ \\
\hline 16. & $\begin{array}{l}\text { Why should I demand for } \\
\text { Nokia? }\end{array}$ & $\mathrm{x}$ & $\mathrm{x}$ & $\mathrm{x}$ & $\mathrm{x}$ & $\mathrm{x}$ \\
\hline 17. & Why should I call for Nokia? & $\sqrt{ }$ & $\mathrm{x}$ & $\mathrm{x}$ & $\mathrm{x}$ & $\sqrt{ }$ \\
\hline 18. & Why should I require Nokia? & $\sqrt{ }$ & $\mathrm{x}$ & $\mathrm{x}$ & $\mathrm{x}$ & $\sqrt{ }$ \\
\hline 19. & Why should I want Nokia? & $\mathrm{x}$ & $\mathrm{x}$ & $\sqrt{ }$ & $\mathrm{x}$ & $\sqrt{ }$ \\
\hline 20. & Why should I prefer Nokia? & $\sqrt{ }$ & $\mathrm{x}$ & $\sqrt{ }$ & $\sqrt{ }$ & $\sqrt{ }$ \\
\hline 21. & Why should I desire for Nokia? & $\sqrt{ }$ & $\mathrm{x}$ & $\sqrt{ }$ & $\mathrm{x}$ & $\sqrt{ }$ \\
\hline 22. & Why should I opt for Nokia? & $\mathrm{x}$ & $\mathrm{x}$ & $\mathrm{x}$ & $\mathrm{x}$ & $\sqrt{ }$ \\
\hline 23. & Why should I pick Nokia? & $\mathrm{x}$ & $\mathrm{x}$ & $\mathrm{x}$ & $\mathrm{x}$ & $\mathrm{X}$ \\
\hline 24. & Why should I select Nokia? & $\mathrm{x}$ & $\mathrm{x}$ & $\mathrm{x}$ & $\mathrm{x}$ & $\sqrt{ }$ \\
\hline 25. & Why should I wish for Nokia? & $\mathrm{x}$ & $\mathrm{x}$ & $\sqrt{ }$ & $\mathrm{x}$ & $\sqrt{ }$ \\
\hline 26. & Why should I aspire for Nokia? & $\sqrt{ }$ & $\sqrt{ }$ & $\sqrt{ }$ & $\sqrt{ }$ & $\sqrt{ }$ \\
\hline 27. & Why Nokia is first choice? & $\sqrt{ }$ & $\mathrm{x}$ & $\mathrm{x}$ & $\mathrm{x}$ & $\sqrt{ }$ \\
\hline 28. & $\begin{array}{l}\text { Why I is inclined towards } \\
\text { Nokia? }\end{array}$ & $\sqrt{ }$ & $\mathrm{x}$ & $\sqrt{ }$ & $\mathrm{x}$ & $\sqrt{ }$ \\
\hline 29. & Why should I favor Nokia? & $\sqrt{ }$ & $\sqrt{ }$ & $\sqrt{ }$ & $\sqrt{ }$ & $\sqrt{ }$ \\
\hline 30 & Why should I order Nokia? & $\mathrm{x}$ & $\mathrm{x}$ & $\mathrm{x}$ & $\mathrm{x}$ & $\mathrm{x}$ \\
\hline 31. & Why should I insist for Nokia? & $\mathrm{x}$ & $\mathrm{x}$ & $\sqrt{ }$ & $\mathrm{x}$ & $\sqrt{ }$ \\
\hline 32. & Why should I neglect Nokia? & $\sqrt{ }$ & $\sqrt{ }$ & $\sqrt{ }$ & $\sqrt{ }$ & $\sqrt{ }$ \\
\hline 33. & $\begin{array}{l}\text { Why should I stop thinking } \\
\text { about Nokia? }\end{array}$ & $\sqrt{ }$ & $\mathrm{x}$ & $\mathrm{x}$ & $\mathrm{x}$ & $\sqrt{ }$ \\
\hline 34. & $\begin{array}{l}\text { Why should I put Nokia out of } \\
\text { his mind? }\end{array}$ & $\mathrm{x}$ & $\mathrm{x}$ & $\mathrm{x}$ & $\mathrm{x}$ & $\mathrm{x}$ \\
\hline 35. & $\begin{array}{l}\text { Why should I feel cheated in the } \\
\text { end? }\end{array}$ & $\mathrm{x}$ & $\mathrm{x}$ & $\sqrt{ }$ & $\sqrt{ }$ & $\sqrt{ }$ \\
\hline 36. & Why should I be happy? & $\sqrt{ }$ & $\mathrm{x}$ & $\sqrt{ }$ & $\sqrt{ }$ & $\sqrt{ }$ \\
\hline 37. & $\begin{array}{l}\text { Why should I feel satisfied } \\
\text { finally? }\end{array}$ & $\sqrt{ }$ & $\sqrt{ }$ & $\sqrt{ }$ & $\sqrt{ }$ & $\sqrt{ }$ \\
\hline 38. & Why should one leave Nokia? & $\sqrt{ }$ & $\mathrm{x}$ & $\mathrm{x}$ & $\mathrm{x}$ & $\sqrt{ }$ \\
\hline 39. & Why should one love Nokia? & $\sqrt{ }$ & $\mathrm{x}$ & $\sqrt{ }$ & $\sqrt{ }$ & $\sqrt{ }$ \\
\hline
\end{tabular}


Table 3. Accuracy of different methods [23].

\begin{tabular}{|c|c|c|c|c|c|}
\hline Method & Method 1 & Method 2 & Method 3 & Method 4 & Our M ethod \\
\hline Accuracy & 0.64 & 0.23 & 0.53 & 0.33 & 0.87 \\
\hline
\end{tabular}

\section{Results and Discussions}

We analyze the results and get following observations. We find that our proposed Method gives maximum accuracy of $60 \%$ in segmentation of Why-questions in view for opinion mining. We re-computes sentiment scores of words to give updated positive and negative scores and determine sentiment polarity of WHY type questions. The computed scores of words through our algorithm exhibit better results with maximum accuracy of 0.87 than the scores assigned to the words in SentiWordNet, MPQA, WordNet, and Bing Liu's Opinion Lexicon in determining sentiment polarity of WHY questions.

1. WSD (word sense disambiguation) - we calculate the average sum of all scores of the word related to a given part of speech in SentiWordNet. Words behave differently in terms of polarity in different context. Hence identification of the word sense and allotting the score of the sense directly could improve the performance of the systems. Such as Why I need camera $x$ ? Here, average sum of need word leads to negative polarity.

2. Opinion bearing words- identification of opinion bearing words in the sentence could increase the performance of the proposed system. Our system calculates the scores of all words of the sentences.

3. Discourse analysis - we use PDTB-Styled End-to-End Discourse Parser developed by Ziheng Lin et al. [45] as the accuracy of discourse parser in today's era is not very promising hence it affect our performance.

4. Domain specific lexicon. SentiWord Net, MPQA, Bing Liu lexicon are open domain dictionary. Some domain specific lexicons behave differently in polarity than general domain lexicons. E.g. long. If the camera coverage is long then it is good. But the movie is long it expresses negative sentiments.

5. Informal language. Use of informal language effect the method.

6. Use of knowledge-based techniques for opinion mining- we find from literature [29, 30, 31, 32, 33] that the bag of concept model captures conceptual and affective information and are more suitable for task of opinion mining. We will consider using the same in future and investigate the worthiness of them in determining sentiment polarity of whyquestions. 


\section{Conclusions and Future Works}

In this paper, we determine the polarity of the questions that could be single or multiple sentence(s) why-type questions through proposed algorithm. We perform discourse based analysis of why type questions before computing sentiment polarity of question through average scoring method. The segmentation of why-questions and their sentiment determination are dependent on performance of automatic discourse parser. Instead of calculating score for all words, we observe that detecting opinion bearing words and computing their sentiment scores could improve the performance of why-QAS. We know SentiWord Net, MPQA is general domain dictionary hence there should be domain specific learning to use same. We find that requirements of people depend upon their choice, age, time, financial status. Hence capturing their requirements from their browsing history as in recommender systems then presenting good or bad quality of the product or services [36] will be more good option. In future we will use different discourse parsers, patterns, i.e., sentic patterns [34, 35] or textual [37] entailment system, semis-supervised [38] learning to evaluate and compare our methods on different parameters. We will use machine learning methods for the task of sentiment polarity detection of questions as it could be effective in different domains.

\section{References}

1. Hongping Fu et al.: Classification of opinion questions. In Proceedings of the 35th ECIR conference, Moscow (2013)

2. Jong-Hoon Oh et al.: Why-question answering using sentiment analysis and word classes. In Proceedings of EMNLP-CoNLL, Korea (2012)

3. Chihli Hung, Hao-Kai Lin: Using Objective Words in SentiWordNet to improve Sentiment Classification for Word of Mouth. IEEE Intelligent Systems 28(2), 47-54 (2013)

4. S. Moghaddam and M. Ester: AQA: Aspect-based Opinion Question Answering. In IEEEICDMW, Vancouver, Canada (2011)

5. Yu J, Zha Z-J, Wang M, Chua T-S: Answering opinion questions on products by exploiting hierarchical organization of consumer reviews. In Proceedings of EMNLP conference, Jeju, Korea (2012)

6. L.W. Ku, Y.T. Liang, et al.: Question Analysis and Answer Passage Retrieval for Opinion Question Answering Systems. International Journal of Computational Linguistics \& Chinese Language Processing (2007)

7. T. Wilson, J. Wiebe et al.: Recognizing Contextual Polarity in Phrase-level Sentiment Analysis. In HLT/EMNLP (2005)

8. S. Moghaddam and F. Popowich: Opinion polarity identification through adjectives. CoRR, abs/1011.4623 (2010)

9. Esuli and F. Sebastiani: SentiWordNet: A publicly available lexical resource for opinion mining. In Proceedings of LREC-06, the 5th Conference on Language Resources and Evaluation, Geneva, Italy (2006)

10. Stanford Part of Speech Tagger: http://nlp.stanford.edu/software/tagger.shtml

11. Gautam Kumar et al.: Opinion mining and summarization for customer reviews. IJEST Volume 4, Issue 8 (2012) 
12. Shaishav Agrawal: Feature based Star Rating of Reviews: A Knowledge-Based Approach for Document Sentiment Classification. International Journal of Hybrid Information Technology, Vol. 5, No. 4 (2012)

13. M. Abulaish, Jahiruddin, M.N. Doja, T. Ahmad: Feature and Opinion Mining from Customer Review Documents. In Proceedings of Pattern Recognition and Machine Intelligence (2009)

14. V. Hatzivassiloglou and K. R. McKeown: Predicting the semantic orientation of adjectives. In Proc. of ACL (1998)

15. C. Fellbaum (ed.): Word Net: An Electronic Lexical Database. MIT Press (1998)

16. Farah Benamara, Carmine Cesarano, Diego Reforgiato: Sentiment Analysis: Adjectives and Adverbs are better than Adjectives Alone. In Proc. of ICWSM "Boulder, CO USA

17. Turney, P.: Thumbs up or thumbs down? Semantic orientation applied to unsupervised classification of reviews. In Proceedings of the 40th Annual Meeting of the Association of Computational Linguistics (2002)

18. Sentiment Symposium Tutorial: Lexicons. http://sentiment.christopherpotts.net/lexicon/ (June, 2013)

19. Bing Liu: A list of positive and negative opinion words or sentiment words for English, http://www.cs.uic.edu/ liub/FBS/opinion-lexicon-English.rar, last accessed on 11th June, 2013.

20. K. Denecke: Using SentiWordNetfor Multilingual Sentiment Analysis. In Proceedings of the International Conference on Data Engineering (ICDE 2008), Workshop on Data Engineering for Blogs, Social Media, and Web 2.0, Cancun (2008)

21. Ziheng Lin et al.: A PDTB-Styled End-to-End Discourse Parser. http://wing.comp.nus.edu.sg/ linzihen/parser/

22. Fan Bu: Function-based question classification for general QA. In Proceedings of the Conference on Empirical Methods in Natural Language Processing, pp. 1119-1128, Massachusetts, USA (2010)

23. S Padmaja et al.: Opinion Mining and Sentiment Analysis - An Assessment of Peoples' Belief: A Survey. International Journal of Adhoc, Sensor \& Uboquitos Computing, Volume 4; Issue 1 (2013)

24. Liu, Y., Li, S., Cao, Y., Lin, C.-Y., Han, D., \& Yu, Y.: Understanding and summarizing answers in community-based question answering services. In Proceedings of the 22nd International Conference on Computational Linguistics (COLING 2008), pp. 497-504, Stroudsburg, PA (2008)

25. B. Heerschop et al.: Polarity Analysis of Texts Using Discourse Structure. In Proc. 20th ACM Intl. Conf. Information and Knowledge Management, ACM, pp. 1061-1070 (2011)

26. R. Higashinaka and H. Isozaki: Corpus-based Question Answering for "why"e -Questions. In Proceedings of the International Joint Conference on Natural Language Processing (IJCNLP), pages 198-425 (2008)

27. S. Verberne, L. Boves, N. Oostdijk, and P.A. Coppen: What is not in the Bag of Words for "why"-QA? Computational Linguistics (2010)

28. Opinion Finder : http://mpqa.cs.pitt.edu/opinionfinder/

29. S. Poria, A. Gelbukh, A. Hussain, N. Howard, D. Das, S. Bandyopadhyay: Enhanced SenticNet with Affective Labels for Concept-based Opinion Mining. IEEE Intelligent Systems, vol. 28, issue 2 (2013)

30. S. Poria, A. Gelbukh, E. Cambria, P. Yang, A. Hussain, T. Durrani: Merging SenticNet and WordNet-Affect emotion lists for sentiment analysis. In IEEE 11th International Conference on Signal Processing, IEEE ICSP 2012, China, Vol. 2, pp. 1251-1255 (2012) 
31. S. Poria, A. Gelbukh, E. Cambria, D. Das, S. Bandyopadhyay: Enriching SenticNet Polarity Scores through Semi-Supervised Fuzzy Clustering. In Workshop on Sentiment Elicitation from Natural Text for Information Retrieval and Extraction, SENTIRE 2012, IEEE 12th International Conference on Data Mining Workshops (ICDMW), Belgium, IEEE CS Press, pp. 709-716 (2012)

32. S. Poria, A. Gelbukh, D. Das, S. Bandyopadhyay: Fuzzy Clustering for Semi-Supervised Learning-Case study: Construction of an Emotion Lexicon. Lecture Notes in Artificial Intelligence, N 7629, pp. 73-86 (2012)

33. Poria, S., Agarwal, B., Gelbukh, A., Hussain, A., Howard, N.: Dependency-Based Semantic Parsing for Concept-Level Text Analysis. In Computational Linguistics and Intelligent Text Processing, pp. 113-127, Springer (2014)

34. S. Poria, E. Cambria, G. Winterstein, and G.-B. Huang. Sentic patterns: Dependencybased rules for concept-level sentiment analysis. Knowledge-Based Systems 69, pp. 45-63 (2014)

35. S. Poria, A. Gelbukh, E. Cambria, A. Hussain, and G.-B. Huang: EmoSenticSpace: A novel framework for affective common-sense reasoning. Knowledge-Based Systems, 69, pp. 108-123 (2014)

36. S. Poria, E. Cambria, L.-W. Ku, C. Gui, A. Gelbukh: A rule-based approach to aspect extraction from product reviews. In: COLING, Dublin (2014)

37. Pakray, P., Neogi, S., Bhaskar, P., Poria, S., Bandyopadhyay, S., \& Gelbukh, A.: A Textual Entailment System using Anaphora Resolution. System Report. Text Analysis Conference Recognizing Textual Entailment Track Notebook (2011)

38. Poria, S., Gelbukh, A., Hussain, A., Bandyopadhyay, S., Howard, N.: Music genre classification: A semi-supervised approach. Pattern Recognition, pp. 254-263, Springer (2013) 